\title{
Letter to the Editor: “Integration of PET/ CT Radiomics and Semantic Features for Differentiation Between Active Pulmonary Tuberculosis and Lung Cancer"
}

\author{
Donghui Hou' ${ }^{1}$, and Tingting $\mathrm{Cui}^{2}{ }^{2}$ \\ ${ }^{1}$ Department of Diagnostic Radiology, National Cancer Center/National Clinical Research Center for Cancer/Cancer Hospital, Chinese \\ Academy of Medical Sciences and Peking Union Medical College, Beijing, China \\ ${ }^{2}$ Department of Radiology, Cancer Hospital of China Medical University/ Liaoning Cancer Institute \& Hospital, No.44, Xiaoheyan \\ Dadong, Shenyang 110801, China 2021
}

We read with great interest the research article "Integration of PET/CT Radiomics and Semantic Features for Differentiation between Active Pulmonary Tuberculosis and Lung Cancer" [1]. We applaud Dr. Du and the other researchers for the article on differential diagnosis between active pulmonary tuberculosis (TB) and lung cancer (LC). At present, lung cancer has become the disease with the highest morbidity and mortality globally, and pulmonary TB is one of the leading causes of death in infectious diseases. It is still a threat to global public health, especially in developing countries.

However, we have several questions and comments, which focus on materials and methods. First, as a chronic infectious disease that can cause inflammatory damage and pulmonary fibrosis, pulmonary TB increases the risk of LC. Meanwhile, immunosuppression induced by $\mathrm{LC}$ and chemotherapy may also increase the incidence of pulmonary TB $[2,3]$. Pulmonary TB complicated with LC is becoming more and more common. Some lesions consist of pulmonary TB and LC, and needle biopsy or bronchoscopy biopsy may not find two components. In which cases, we misdiagnose them as pulmonary TBs or LCs, which results in inaccuracy in raw data. Do you think it is necessary to exclude the subjects that confirmed by small biopsy specimens? Second, as far as we know, some lesions may not be sent for frozen pathological examination when surgeons observe typical manifestations of pulmonary TB during operations such as caseous necrosis, pus. If the lesions consist of pulmonary TB and LC, the lesions will be misdiagnosed as pulmonary TB, and the entire series is flawed. Can the authors expound on this as well? Next, the inclusion criteria include solid lesions, but the authors do not clarify the

Correspondence to: Tingting Cui; e-mail: 2515043212@qq.com specific standards. In addition, why do the authors exclude the cystic-solid lesions, the authors also did not explain.

Moreover, we notice that the authors chose an experienced nuclear medicine physician to outline all the subjects' the regions of interest (ROI). Subsequently, they randomly selected thirty patients in the training cohort, and another expert nuclear medicine physician evaluated inter-observer reproducibility of radiomics analysis, which is reasonable. However, the authors did not mention the outcome of reproducibility in the two processes. Besides, this is a single institution analysis without external validation set. How is the reproducibility of the radiomics nomogram developed in this study? Can they elaborate on these?

\section{Declarations}

Conflict of Interest The authors declare that they have no conflict of interest.

\section{References}

1. Du D, Gu J, Chen X et al (2021) Integration of PET/CT radiomics and semantic features for differentiation between active pulmonary tuberculosis and lung cancer. Mol Imaging Biol 23:287-298

2. Engels EA (2008) Inflammation in the development of lung cancer: epidemiological evidence. Expert Rev Anticancer Ther 8:605-615

3. Wu CY, Hu HY, Pu CY et al (2011) Pulmonary tuberculosis increases the risk of lung cancer: a population-based cohort study. Cancer 117:618-624

Publisher's Note Springer Nature remains neutral with regard to jurisdictional claims in published maps and institutional affiliations. 